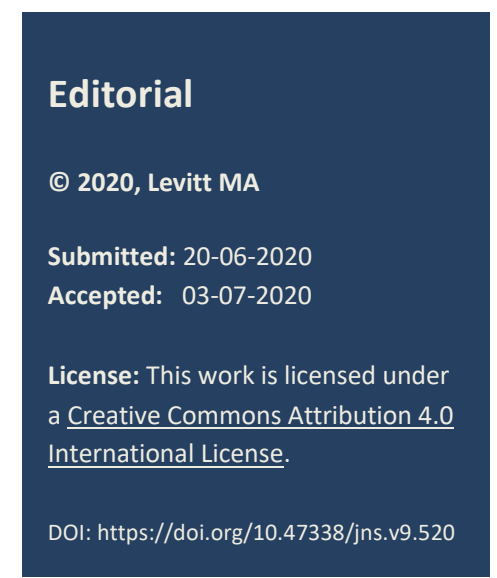

\section{Where are we in pediatric colorectal and pelvic reconstructive surgery? New insights and the future}

\author{
Marc A. Levitt*
}

Division of Colorectal and Pelvic Reconstructive Surgery, Children's National Hospital, 111 Michigan Avenue, Washington DC 20010 USA

Correspondence*: Marc A. Levitt, MD, Chief, Division of Colorectal and Pelvic Reconstructive Surgery, Children's National Hospital, 111 Michigan Avenue, Washington DC 20010 USA,

E-mail: mlevitt@childrensnational.org

\section{Introduction}

A popular children's book, Everyone Poops, by Taro Gomi, demonstrates that the physiology of stooling is thought about, makes children and parents concerned, and is a focus of much of a child's early development. The book has deep meaning, particularly when one recognizes that there is a follow-up to this book - Pooh Gets Stuck.

References to pediatric colorectal problems go back many thousands of years. In fact, in the Babylonian Talmud written in 200 A.C., the following treatment is described: "An infant whose anus is not visible should be rubbed with oil and stood in the sun...where it shows transparent it should be torn crosswise with a barley grain". Our surgical techniques to manage such patients have certainly evolved since that time.

For those interested in the field of pediatric colorectal surgery, our desire is to help children in whom "poo gets stuck' (in all of its forms). We hope in our clinics, wards, and operating rooms, to improve the lives of as many children as possible.

My personal journey in this field began as medical student, in 1992; I signed up for an elective in pediatric surgery and met Alberto Pena, one of the pioneers in the field of colorectal care (Fig. 1). This changed my career trajectory in a very positive way. I observed Dr. Pena providing all aspects of care to these patients. However, as I advanced in my training it seemed increasingly complex and knew I needed help from collaborators.

The modern pediatric colorectal story for anorectal malformations began in Melbourne, Australia when Douglas Stephens worked on defining the anatomy of children with anorectal malformation. He did this by diligently performing autopsies on patients with these conditions. Prior to this, the anatomy of such patients was only a concept that existed in surgeons' minds. The anatomy was believed to look like the images shown in Figure 2, in the bible of pediatric surgery in North America, the Gross textbook, which in retrospect was both oversimplified and inaccurate.

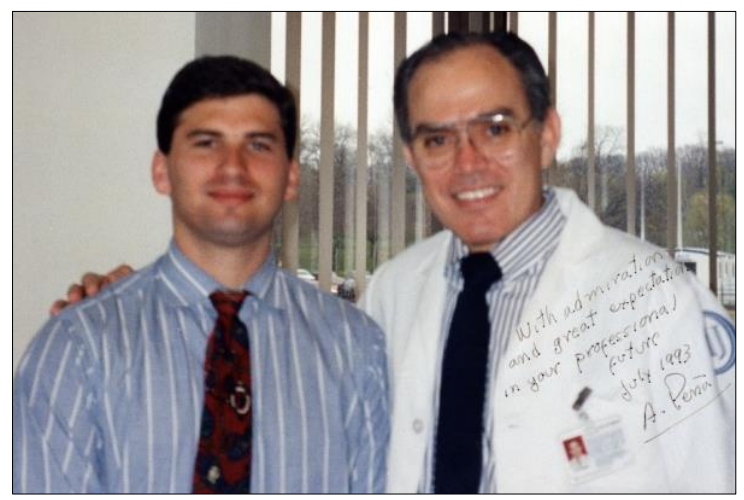

Figure 1: Marc Levitt and Alberto Pena in 1993.

Dr. Stephens during his autopsy dissections came to several anatomic conclusions, most notably that a sphincter mechanism surrounded the rectum, the puborectalis sling. The surgery he proposed, the Stephens technique, involved a perineal dissection to find a path for the distal rectum to be pulled through this sling (Fig. 3). Justin Kelly, one of Stephen's trainees in Australia learned how to do this, and then travelled to Boston for further training. At Boston Children's Hospital, he taught what he had learned from Stephens to the surgeons there, including another trainee, Alberto Pena. After completing his training in Boston, Pena went to Mexico City in 1972 to become the head of surgery at the National Pediatric Institute. When he asked his new partners to choose an area of specialization, no one choose colorectal, so he took on that group of patients and embarked on his revolutionary colorectal career. Pena at first applied the Stephens technique to children with anorectal malformations but he became increasingly frustrated 
by the procedure as it was blind and had very poor exposure. Over time his incision grew longer and longer. This culminated in 1980 thanks to a collaboration with Dr. Peter Devries who had come to Mexico City to work on these cases with Pena, with the first posterior sagittal anorectoplasty.

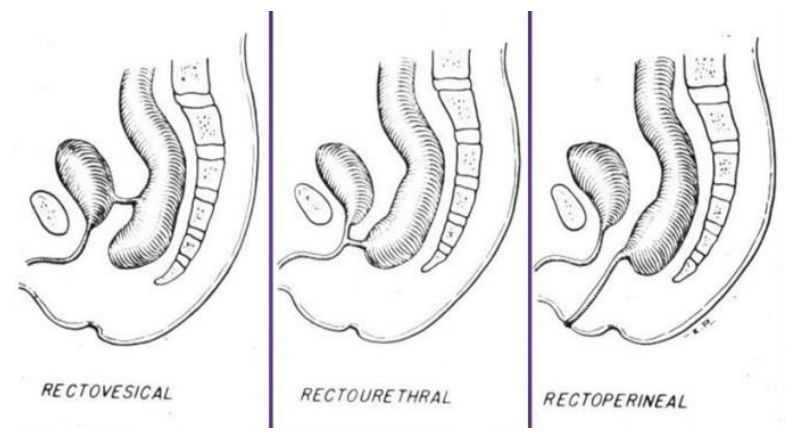

Figure 2: Gross and Ladd Textbook, Drawings of the anatomy of anorectal malformations.

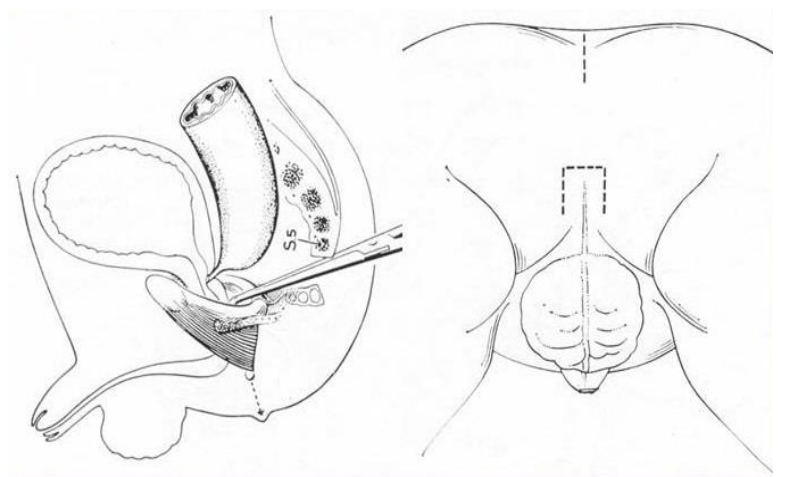

Figure 3: Stephen's sacroperineal approach, 1953.

This posterior sagittal approach opened a Pandora's Box. It allowed for a true understanding of the pelvic anatomy and led to the care of many conditions, previously, to use Pena's description, very difficult to reach from above (via laparotomy) and very difficult to reach from below (perineally). The approach influenced the repair of cloacal malformations, urogenital sinus, pelvic tumors, urethral problems, reoperations for imperforate anus and for Hirschsprung disease, a transpubic approach (splitting the pubis for complex genitourinary problems inaccessible any other way), and a comprehensive strategy for the management of cloacal exstrophy. Perhaps the greatest contribution that came from a focused approach to these patients was nonsurgical, namely development of the concept of bowel management for fecal incontinence. Thanks to such programs, thousands of children are no longer in diapers and have said good-bye to their stomas, an impact perhaps comparable to the use of intermittent catheterization for children with urinary incontinence.

During these years, it was clear that myriad concerns related to patients' urologic, gynecologic, and GI/motility systems exist demanding a new paradigm of care. I saw the care of such children being done well by a single clinician, and simultaneously witnessed the complexity of medicine growing. I also realized that the care of children with colorectal problems is very difficult and harder than most realize. It requires an integrated and collaborative care as all the anatomic structures lie right next to each other (Fig. 4).

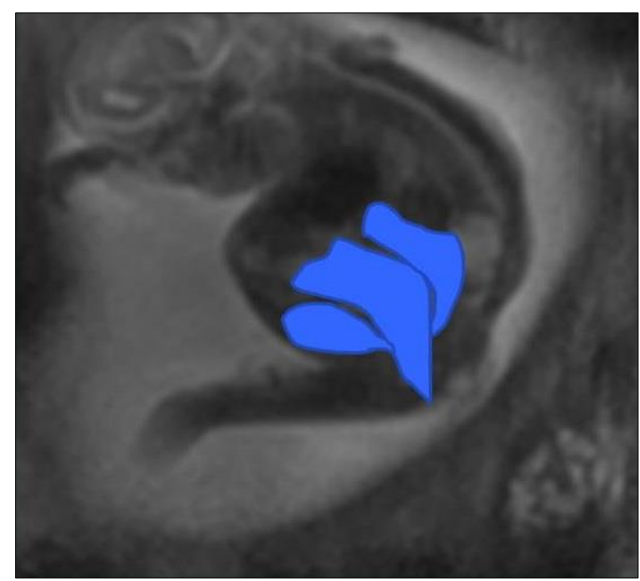

Figure 4: Fetal MRI of a 22 week fetus with a cloaca, and the bladder, vagina and rectum are highlighted in blue.

To achieve success, patients with anorectal malformations (ARM), Hirschsprung disease (HD), fecal incontinence from a variety of conditions, and colonic motility disorders requires care from specialists across a variety of fields throughout their lives. These include colorectal surgery, urology, gynecology, and GI motility, as well as orthopedics, neurosurgery, anesthesia, pathology, radiology, psychology, social work, nutrition, amongst many others. Perhaps most important though, to their achievement of a good functional result, is their connection to superb nursing care.

Having met many parents with newborns diagnosed with colorectal problems I have made several observations. First, no parent seems to have ever thought that their child could have a problem with stooling - this is a physiologic ability that is taken for granted, and when told this was a problem, they are usually shocked that something like this could happen. Second, when discussing with them that their child will need surgery to correct their colorectal anatomy, none focus on the surgical technique and elegance of the anal reconstruction, as I do. All instead focus on whether that technique will create an anatomy that will work, and will allow their child to stool without difficult, and become socially normal. As surgeons we need to remember this - we always need to understand what it is that the family and patient wishes for us to deliver to them. As proud of our surgical skills as we are, it is the functional outcome that matters most.

I like to say that a complex colorectal operation takes about four hours to perform, but in order to get a good result takes an additional 96 hours of work - the vast majority of which involves nursing care. A nurse's 
skills in identifying problems, solving them, and always striving to fill the gaps, are unique. I am so convinced, and often shout from the rooftops, that without my nursing partners I would have achieved very little as a surgeon.

With a collaborative model we can achieve great things, and we need to always remember that "it is not the unanswered questions, but rather the unquestioned answers that one must pursue."

A few such advances have included:

- Work in accurate prenatal diagnosis of anorectal and cloacal malformations

- Management of the complex newborn, particularly improvements in newborn radiology and neonatal care

- Recognizing and treating associated urologic anomalies

- Recognizing and treating associated gynecologic concerns

- Developing a protocol to predict continence, even in the newborn period

- Surgical interventions of the newborn and knowing when a primary repair vs. a staged approach is appropriate

- Defining of anatomy so patients can be compared across centers, and treatment options and outcomes are uniformly analyzed

- Recognizing the value of laparoscopy and knowing for which cases this approach should be applied

- Development of a treatment algorithm for management of cloacal malformations taking into account the importance of their common channel and urethral lengths

- Recognizing key complications after ARM and Hirschsprung surgery, knowing when and how to do a reoperation, determining the outcomes of such reoperations, and ultimately figuring how to avoid complications altogether

- Learning the physiology of fecal continence, which patients suffer from it, and the predictors of continence

- Development of a bowel management program and committing to following patients long term

- Recognizing the vital collaboration with GI/motility and offering surgical adjuncts to treatment, when appropriate, including resection and antegrade options

- Learn the pathophysiology of motility disorders, and based on a better understanding of this, develop treatment protocols

- Collaborating with urology to render a patient clean and dry for both urine and stool and knowing when the colon could be used for a bladder augment, or if the appendix can be shared between Malone and Mitrofanoff

- Developing of new technologies, such as sacral nerve stimulation, and understanding for which patients it is appropriate

- Development of dedicated center, that is integrated and collaborative and documenting that such a center provides better outcomes

- Recognizing the range of ages we need to care for and establishing transition program for adults

- Developing research protocols to define the genetics of these disorders and working toward tissue engineering of structures that congenitally have failed to correctly develop

- Using strategies to have real time data, follow outcomes, and respond to changes

- Development of international consortiums that work well together to help patients in a way not achievable by a single institution

- Bringing complex care to all corners of the world including to the developing world

- Reaffirming the key principles stated by Sir Dennis Browne, to help set a standard of care and not seek a monopoly

It is vital that caregivers understand the daily struggle of improving a patient's quality of life, and that they learn the key skills and tricks to achieve good results. If they accomplish this lofty goal they will feel very gratified.

Conflict of Interest: None declared

Source of Support: Nil

Consent to Publication: Author(s) declared taking informed written consent for the publication of clinical photographs/material (if any used), from the legal guardian of the patient with an understanding that every effort will be made to conceal the identity of the patient, however it cannot be guaranteed.

Author Contributions: Author(s) declared to fulfil authorship criteria as devised by ICMJE and approved the final version. 